\title{
ON LINKED BINARY REPRESENTATIONS OF PAIRS OF INTEGERS: SOME THEOREMS OF THE ROMANOV TYPE ${ }^{1}$
}

\author{
BY G. J. RIEGER
}

Communicated by P. T. Bateman, March 7, 1963

1. Introduction. Let us denote by $N$ the sequence $\{1,2,3, \cdots\}$, by $p$ a prime, by $(a, b)$ the greatest common divisor of $a$ and $b$, by $[a, b]$ the least common multiple of $a$ and $b$, by $\{*: \cdots\}$ resp. $A\{*: \cdots\}$ the set resp. number of $*$ with the properties $\cdots$, by $\mu$ the Moebius function, by $C$ an absolute positive constant and by $C\left(^{*}\right)$ a positive constant depending on * only.

Suppose $N_{j} \subset N(j=1,2,3,4)$ and denote by $y_{1} \sim y_{2}$ an arbitrary relation (= linking) with $y_{1,2} \in N$. For instance, $\left[y_{1} \sim y_{2}\right]$ : $=\left[\left(y_{1}, y_{2}\right)=1\right]$ resp. $\left[y_{1} \sim y_{2}\right]:=\left[y_{1}=y_{2}\right]$ can be considered a weak resp. strong linking. By a linked binary representation of a pair $m, n$ with $m \in N$ and $n \in N$ we mean a solution $x_{1}, x_{2}, x_{3}, x_{4}$ of the Diophantine system $x_{1}+x_{2}=m \wedge x_{3}+x_{4}=n \wedge x_{j} \in N_{j}(j=1,2,3,4) \wedge x_{2} \sim x_{4}$. Various generalizations are obvious (more summands, triples, etc.). We do not intend to give a detailed and general study of the questions arising in this context. We rather prefer to investigate two special problems of this type with $\sim$ being $=$; they are inspired by the following two well-known results of Romanov:

$$
E_{a}:=\left\{m: m=p+v^{a} \wedge p \text { prime } \wedge v \in N\right\} \quad(1<a \in N)
$$

and

$$
F_{a}:=\left\{m: m=p+a^{v} \wedge p \text { prime } \wedge v \in N\right\} \quad(a \in N)
$$

have positive asymptotic density [1, pp. 63-70].

2. On Romanov's first theorem. Generalizing the result for $E_{a}$, we show that the set $\left\{m, n: m=p_{1}+v^{a} \wedge n=p_{2}+v^{a} \wedge p_{1,2}\right.$ prime $\left.\wedge v \in N\right\}$, considered as a set of lattice points in the plane, has positive asymptotic density in the plane:

ThEOREM 1. For $1<a \in N$ there exist constants $C_{1}(a)$ and $C_{2}(a)$ such that $x>C_{1}(a)$ implies

$$
\begin{aligned}
& A_{1}(x, a):=A\{m, n: m<x \wedge n<x \wedge m=p_{1}+v^{a} \wedge \\
&\left.n=p_{2}+v^{a} \wedge p_{1,2} \text { prime } \wedge v \in N\right\}>C_{2}(a) x^{2} .
\end{aligned}
$$

1 With support from NSF grant G-16305 to Purdue University. 
Proof. Let $f_{1}(m, n ; a):=A\left\{p_{1}, p_{2}, v: p_{1}+v^{a}=m \wedge p_{2}+v^{a}=n\right\} ;$ since $A_{1}(x, a)=A\left\{m, n: m<x \wedge n<x \wedge f_{1}(m, n ; a)>0\right\}$, the Schwarz inequality yields

$$
\left(\sum_{m<x} \sum_{n<x} f_{1}(m, n ; a)\right)^{2} \leqq A_{1}(x, a) \sum_{m<x} \sum_{n<x} f_{1}^{2}(m, n ; a) .
$$

On the one hand, we find

$$
\sum_{m<x} \sum_{n<x} f_{1}(m, n ; a)=A\left\{p_{1}, p_{2}, v: p_{1}+v^{a}<x \wedge p_{2}+v^{a}<x\right\}
$$

$$
\begin{array}{ll}
\geqq A\left\{p_{1}: p_{1}<\frac{x}{2}\right\} A\left\{p_{2}: p_{2}<\frac{x}{2}\right\} A\left\{v: v^{a}<\frac{x}{2}\right\} \\
>C_{4}\left(\frac{x}{\log x}\right)^{2}\left(\frac{x}{2}\right)^{1 / a} & \left(x>C_{3}(a)\right) .
\end{array}
$$

On the other hand, we find

$$
\begin{aligned}
S_{1}(x, a): & =\sum_{m<x} \sum_{n<x} f_{1}^{2}(m, n ; a) \\
& =A\left\{p_{1}, p_{2}, p_{3}, p_{4}, v_{1}, v_{2}: p_{1}+v_{1}^{a}=p_{2}+v_{2}^{a}<x \wedge p_{3}+v_{1}^{a}\right. \\
& \left.=p_{4}+v_{2}^{a}<x\right\} \\
& \leqq \sum_{v_{1}<x^{1 / a}} \sum_{v_{2}<x^{1 / a}} A\left\{p_{1}, p_{2}, p_{3}, p_{4}: p_{1}-p_{2}=p_{3}-p_{4}\right. \\
& \left.=v_{2}^{a}-v_{1}^{a} \wedge p_{1,2,3,4}<x\right\} .
\end{aligned}
$$

In case of $v_{1}=v_{2}$ resp. $v_{1} \neq v_{2}$ we use

$$
A\{p: p<x\}<C_{5} \frac{x}{\log x}
$$

resp. Brun's sieve method [2, 2. Satz 4.2] and obtain

$$
S_{1}(x, a)<C_{7}\left(\frac{x}{\log x}\right)^{2} x^{1 / a}+2 \sum_{v_{2}<v_{1}<x^{1 / a}}\left(C_{8} \frac{x}{\log ^{2} x} g\left(v_{1}^{a}-v_{2}^{a}\right)\right)^{2}
$$

where

$$
g(b):=\prod_{p \mid b}\left(1+\frac{1}{p}\right)=\sum_{d \mid b ; \mu(d) \neq 0} \frac{1}{d}
$$

It follows

$$
S_{1}(x, a)<C_{7}\left(\frac{x}{\log x}\right)^{2} x^{1 / a}+C_{9} \frac{x^{2}}{\log ^{4} x} \sum_{u<x} F(u ; x, a) g^{2}(u) \quad\left(x>C_{6}\right)
$$


where

$$
F(u ; x, a):=A\left\{v_{1}, v_{2}: v_{2}<v_{1}<x^{1 / a} \wedge v_{1}^{a}-v_{2}^{a}=u\right\} .
$$

Writing $g(u)$ as a sum and changing the order of summation gives

$$
\sum_{u<x} F(u ; x, a) g^{2}(u)=\sum_{\substack{d_{1}<x \\ \mu\left(d_{1}\right) \neq 0}} \sum_{\substack{d_{2}<x \\ \mu\left(d_{2}\right) \neq 0}} \frac{1}{d_{1} d_{2}} B\left(\left[d_{1}, d_{2}\right] ; x, a\right)
$$

where

$$
B(k ; x, a):=\sum_{u<x ; u \equiv 0 \bmod k} F(u ; x, a)<2 x^{2 / a} k^{-1 / a} a^{w(k)} \quad(\mu(k) \neq 0)
$$

$[1$, p. 66] with

$$
w(k):=A\{p: p \mid k\}<C_{10} \frac{\log k}{\log \log k} .
$$

Since $\mu\left(d_{1}\right) \neq 0 \wedge \mu\left(d_{2}\right) \neq 0$ imply $\mu\left(\left[d_{1}, d_{2}\right]\right) \neq 0$, we obtain

$$
\begin{array}{r}
S_{1}(x, a)<C_{7} \frac{x^{2+1 / a}}{\log ^{2} x}+\frac{x^{2+2 / a}}{\log ^{4} x} C_{11}(a) \sum_{\substack{d_{1}<x \\
\mu\left(d_{1}\right) \neq 0}} \sum_{\substack{d_{2}<x \\
\mu\left(d_{2}\right) \neq 0}}\left(d_{1} d_{2}\right)^{-1}\left[d_{1}, d_{2}\right]^{-1 / 2 a} \\
\left(x>C_{6}\right) .
\end{array}
$$

Using $\left[d_{1}, d_{2}\right]^{2} \geqq d_{1} d_{2}$, we find

$$
S_{1}(x, a)<C_{12}(a) \frac{x^{2+2 / a}}{\log ^{4} x}
$$

(1), (2), and (3) give the desired result.

It is not difficult to determine a dependence of $C_{1,2}(a)$ on $a$ explicitly. Since $A_{1}(x, a) \leqq A\{m, n: m<x \wedge n<x\}$, Theorem 1 is best possible with respect to the order of magnitude in $x$. Theorem 1 is also correct for $a=1$ but of no interest.

3. On Romanov's second theorem. In a similar way we generalize the result for $F_{a}$ :

Theorem 2. For $1<a \in N$ there exist constants $C_{13}(a)$ and $C_{14}(a)$ such that $x>C_{13}(a)$ implies

$$
\begin{aligned}
A_{2}(x, a):=A\left\{m, n: m<x \wedge n<x \wedge m=p_{1}+a^{v} \wedge\right. \\
\left.n=p_{2}+a^{v} \wedge p_{1,2} \text { prime } \wedge v \in N\right\}>C_{14}(a) \frac{x^{2}}{\log x} .
\end{aligned}
$$

Proof. Let $f_{2}(m, n ; a):=A\left\{p_{1}, p_{2}, v: p_{1}+a^{v}=m \wedge p_{2}+a^{v}=n\right\}$. As 
in the preceding proof, we find

$$
\sum_{m<x} \sum_{n<x} f_{2}(m, n ; a)>C_{16}\left(\frac{x}{\log x}\right)^{2} \frac{\log x / 2}{\log a} \quad\left(x>C_{15}(a)\right)
$$

and

$$
\begin{aligned}
& S_{2}(x, a):=\sum_{m<x} \sum_{n<x} f_{2}^{2}(m, n ; a) \\
&<C_{18}\left(\frac{x}{\log x}\right)^{2} \frac{\log x}{\log a}+2 \sum_{v_{2}<v_{1}<\log x / \log a}\left(C_{8} \frac{x}{\log ^{2} x} g\left(a^{v_{1}}-a^{v_{2}}\right)\right)^{2} \\
&\left(x>C_{17}\right) .
\end{aligned}
$$

For $v_{1}>v_{2}$ we have

$$
g\left(a^{v_{1}}-a^{v_{2}}\right)=g(a) g\left(a^{v_{1}-v_{2}}-1\right) ;
$$

with $h:=v_{1}-v_{2}$ we get

$$
\begin{aligned}
& S_{2}(x, a)<C_{19}(a) \frac{x^{2}}{\log x} \\
& +2\left(C_{8} \frac{x}{\log ^{2} x}\right)^{2} \frac{\log x}{\log a} \sum_{h<\log x / \log a} g^{2}\left(a^{h}-1\right) \quad\left(x>C_{17}\right) .
\end{aligned}
$$

For $(a, d)=1$, let $e(a, d)$ denote the exponent of $a \bmod d$ (i.e., the certainly existing smallest $t \in N$ with $\left.a^{t} \equiv 1 \bmod d\right)$; then $d \mid\left(a^{h}-1\right)$ implies $(a, d)=1 \wedge e(a, d) \mid h$. Therefore,

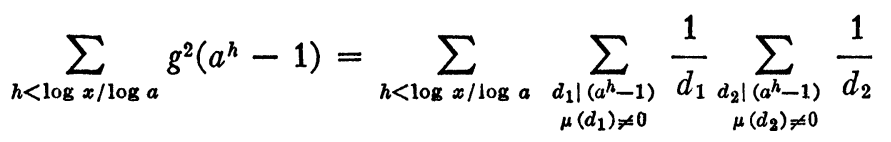

$$
\begin{aligned}
& \leqq \sum_{\substack{d_{1}<x \\
\mu\left(d_{1} \neq 0 \neq 0 \\
\left(d_{1}, a\right)=1\right.}} \sum_{\substack{d_{2}<x \\
\mu\left(d_{2}\right) \neq 0 \\
\left(d_{2}, a\right)=1}} \frac{1}{d_{1} d_{2}} \sum_{\substack{h<\log x / \log a \\
h \equiv 0 \bmod e\left(a, d_{1}\right) \\
h \equiv 0 \bmod e\left(a, d_{2}\right)}} 1 \\
& \leqq \frac{\log x}{\log a} \sum_{\substack{d_{1}<x \\
\mu\left(d_{1}\right) \neq 0 \\
\left(d_{1}, a\right)=1}} \sum_{\substack{\mu\left(d_{2}<x \\
\left(d_{2}\right) \neq 0 \\
\left(d_{2}, a\right)=1\right.}} \frac{1}{d_{1} d_{2}\left[e\left(a, d_{1}\right), e\left(a, d_{2}\right)\right]} \\
& \leqq \frac{\log x}{\log a}\left(\sum_{\substack{d<x \\
\mu(d) \neq 0 \\
(d, a)=1}} d^{-1}(e(a, d))^{-1 / 2}\right)^{2}<C_{20}(a) \log x,
\end{aligned}
$$

since $[a, b]^{2} \geqq a b$ and since, for an arbitrary positive increasing function $f$, 
implies

$$
\sum_{d=1} \frac{1}{d f(d)}<\infty
$$

$$
\sum_{(d, a)=1 ; \mu(d) \neq 0} \frac{1}{d f(e(a, d))}<C_{21}(a, f)
$$

[3, Satz 3]. Hence, we have

$$
S_{2}(x, a)<C_{22}(a) \frac{x^{2}}{\log x}
$$

(4), (5), and (1) with index 2 instead of 1 give the desired result.

It is not difficult to give an explicit dependence of $C_{13}(a)$ and $C_{14}(a)$ on $a$. Again, since

$$
\begin{aligned}
A_{2}(x, a) & \leqq A\left\{p_{1}, p_{2}, v: p_{1,2}<x \wedge a^{v}<x\right\} \\
& <\left(C_{5} \frac{x}{\log x}\right)^{2} \frac{\log x}{\log a}
\end{aligned}
$$

Theorem 2 is best possible in $x$.

4. Generalization to algebraic number fields $K$. For convenience, let $K$ be a totally real algebraic number field. Denote by $n$ the degree of $K$, by $J(K)$ the ring of all integers of $K$, by small Greek letters elements of $J(K)$, by $\xi^{(1)}, \cdots, \xi^{(n)}$ the conjugates of $\xi$, and by $\xi<x$ the system $\left|\xi^{(j)}\right|<x(j=1, \cdots, n) . \pi$ is called a prime if $\pi$ generates a prime ideal of $J(K)$. Combining the method used above with ideas of [4], we arrive at direct generalizations of Theorem 1 and Theorem 2:

TheOREM $1^{\prime}$. For $1<a \in N$ there exist constants $C_{28}(K, a)$ and $C_{24}(K, a)$ such that $x>C_{23}(K, a)$ implies

$$
\begin{gathered}
A\left\{\sigma, \tau: \sigma=\pi_{1}+\nu^{a} \wedge \tau=\pi_{2}+\nu^{a} \wedge \pi_{1,2} \text { prime } \wedge \pi_{1,2} \prec x \wedge \nu \prec x^{1 / a}\right\} \\
>C_{24}(K, a) x^{2 n} .
\end{gathered}
$$

TheOREm 2'. For $0 \neq \alpha \in J(K)$ and not a root of unity there exist constants $C_{25}(K, \alpha)$ and $C_{26}(K, \alpha)$ such that $x>C_{25}(K, \alpha)$ implies $A\left\{\sigma, \tau: \sigma=\pi_{1}+\alpha^{v} \wedge \tau=\pi_{2}+\alpha^{v} \wedge \pi_{1,2}\right.$ prime $\wedge \pi_{1,2}$

$$
\left.\prec x \wedge v \in N \wedge \alpha^{v} \prec x\right\}
$$

$$
>C_{26}(K, \alpha) \frac{x^{2 n}}{\log x} .
$$

Again, the estimates are best possible in $x$. 


\title{
REFERENCES
}

1. E. Landau, Ueber einige neuere Fortschritte der additiven Zahlentheorie, Cambridge Univ. Press, Cambridge, 1937.

2. Karl Prachar, Primzahlverteilung, Springer, Berlin, 1957.

3. G. J. Rieger, Verallgemeinerung eines Sätzes von Romanov und anderes, Math. Nachr. 20 (1959), 107-122.

4. - Verallgemeinerung aweier Sätze von Romanov aus der additiven Zahlentheorie, Math. Ann. 144 (1961), 49-54.

Purdue University and

UNIVERSITY OF MUNICH

\section{THE COHOMOLOGY OF CERTAIN ORBIT SPACES ${ }^{1}$}

\author{
BY P. A. SMITH
}

Communicated by Deane Montgomery, March 11, 1963

Let $(G, X)$ be a topological transformation group-or action-in which $G$ is finite and $X$ is locally compact. An important part of the cohomology of the orbit space $X / G$ lies, so to speak, in the free part $f$ of the action (i.e. the union of orbits of cardinality $[G: 1])$. The cohomology of $\mathrm{f} / G$ can be regarded as an $H(G)$-module. We shall exhibit a complete set of generators and relations for this module assuming $G$ to be the direct product of cyclic groups of prime order $p$ and $X$ to be a generalized sphere over $Z_{p}$ (see $[4$, p. 404]). $H$ will always denote cohomology with values in $Z_{p}$. A useful device consists in relating the generators of $H(G)$ to those of $G$.

Dimension functions. From now on let $G=Z_{p} \times \cdots \times Z_{p}, r$ factors, and let $g_{i}$ be the collection of subgroups of order $p^{i} ; g_{0}$ consists of the identity only. Let $g, h, \cdots$ always denote subgroups of $G$ and $g_{i}, h_{i}, \cdots$ elements of $g_{i}$. In particular $g_{0}=\{1\}$ and $g_{r}=G$.

By a dimension function of the pair $(G, p)$ we shall mean an integervalued function $n(g)$ of constant parity with values $\geqq-1$ and such that for each $g$ different from $G$

$$
n(g)=n(G)+\sum_{h}(n(h)-n(G))
$$

summed over those $h$ 's which lie in $\mathrm{g}_{r-1}$ and contain $g$; when $p=2$, constant parity is not required.

For a given dimension function $n(g)$ let $\Omega$ be the totality of se-

1 This work has been supported by the Office of Naval Research. 